\title{
ON THE INFLUENCE OF HEAT CONDUCTION ON PARAMAGNETIC DISPERSION AND ABSORPTION CURVES
}

\author{
T. P. VALKERING and L. C. VAN DER MAREI, \\ Afdeling der Technische Natuuvkunde ran de Technische Hogeschool Twente, \\ Enschede, Nederland
}

Receiverl 23 October 1969

\section{Synopsis}

Starting from the model of Casimir and Du Pré, which has been refined by Eisenstcin, an expression for the differential paramagnetic susceptibility of a paramagnetic material, placed in a (gaseous or liquid) bath, is derived. This expression contains among others the coefficient of heat conduction and heat capacity of the surroundings and a heat resistance between sample and bath.

Numerical analysis and a short comparison with experiments show that in the temperature region of liquid helium the above-mentioned quantities have a remarkable influence on the dispersion and absorption curves.

1. Intruduclion. In a number of investigations concerning paramagnetic spin-lattice relaxation phenomena ${ }^{1}$ ) the magnetic material is placed in a field of the form

$$
\boldsymbol{H}=\boldsymbol{H}_{\mathbf{c}}+\boldsymbol{h}_{\mathbf{0}} \exp \mathrm{i} \omega t .
$$

$\boldsymbol{H}_{\mathrm{c}}$ is a constant field, $\boldsymbol{H}_{\mathbf{c}} / / \boldsymbol{h}_{\mathbf{0}}$. In the following considerations the sample is assumed to be isotropic and saturation phenomena are neglected. If $\left|\boldsymbol{h}_{0}\right|$ is sufficiently small, the magnetization can be described by

$$
\boldsymbol{M}=\boldsymbol{M}_{\mathbf{c}}+\boldsymbol{m}_{\mathbf{0}} \exp \mathrm{i} \omega t
$$

$\boldsymbol{M}_{\mathrm{c}}$ is the constant part of the magnetization.

As a consequence of relaxation there is a phase difference between

$$
\boldsymbol{m}_{0} \exp \text { i } \omega t \text { and } \boldsymbol{h}_{0} \exp \mathrm{i} \omega t .
$$

To describe this relaxation phenomenon Casimir and Du Pré ${ }^{2}$ ) developed a thermodynamical theory. The essential features of their model are:

A. the magnetic sample is composed of two separate thermodynamic systems:

1. the collection of the magnetic moments (usually called the spin system);

2. the lattice. 
The former is characterized by the state parameters $M, H$ and $T_{\mathrm{s}}$ (spintemperature), the latter by the lattice-temperature $T_{1}$.

B. the encrgy transport from the lattice to the spin-system is given by

$$
\frac{\mathrm{d} Q_{\mathrm{s}}}{\mathrm{d} l}=-\alpha\left(T_{\mathrm{s}}-T_{1}\right) \quad(\alpha>0) .
$$

Supposing that:

1. the specific heat of the lattice is infinitely large,

2. the lattice and the spin system are in internal equilibrium at every moment,

3. the change of the state parameters can be described by a sufficiently small variation around the equilibrium values,

Casimir and Du Pré arrived at the following formula for the reduced differential susceptibility:

$$
\chi \overline{\overline{\mathrm{def}}} \frac{m_{0} / h_{0}}{(\partial M / \partial H)_{T_{\mathrm{s}}}}=1-\frac{F i \omega \tau_{\mathrm{s}}}{1+\mathrm{i} \omega \tau_{\mathrm{s}}},
$$

with $F=\left(C_{H}-C_{M}\right) / C_{H} ; C_{H}$ resp. $C_{M}$ is the specific heat per volume of the spin system at constant $H$ resp. $M ; \tau_{\mathfrak{s}}$, the spin-lattice relaxation time, equals $C_{H} / \alpha$.

Splitting 1.5 into a real and imaginary part $\left(\chi=\chi^{\prime}-\mathrm{i} \chi^{\prime \prime}\right)$ one finds from 1.5 formulae for the dispersion $\chi^{\prime}$ and the absorption $\chi^{\prime \prime}$ :

$$
\left.\begin{array}{l}
\chi^{\prime}=1-F+\frac{F}{1+\omega^{2} \tau_{\mathrm{s}}^{2}} \\
\chi^{\prime \prime}=\frac{F \omega \tau_{\mathrm{s}}}{1+\omega^{2} \tau_{\mathrm{s}}^{2}} .
\end{array}\right\}
$$

Usually $\chi^{\prime}$ and $\chi^{\prime \prime}$ are plotted as functions of ${ }^{10} \log \omega$ (called resp. dispersion and absorption curve).

It has been found that in the temperature region of liquid helium in many cases the experimental results cannot be described by this formulation 3,4 ). Several efforts have been made to give an explanation of this discrepancy. In some considerations the sample is assumed to consist of more than two thermodynamic systems between which heat exchange is possible. In literature ${ }^{5}$ ) are mentioned $e . g$. the spin system, the lattice vibrations on speaking terms with the spin system, and the lattice. Another possibility is to subject the assumptions mentioned in section 1 (1.4) to a critical consideration or one may look for disturbing influences.

If one maintains the idea of two thermodynamic systems, the principal object of the experiments is to find the value of the spin-lattice relaxation 
time. From the experimental dispersion and absorption curves ( $\chi^{\prime}$ resp. $\chi^{\prime \prime}$ vs. $\left.{ }^{10} \log \omega\right)$ quantities with the dimension of time are derived. Some of these quantities are:

$\tau_{\frac{1}{2}}$ : the reciprocal frequency for which $\chi^{\prime}=1-\frac{1}{2} F$,

$\tau_{\text {diss }}$ : the reciprocal frequency for which the dispersion curve has a point of inflection,

$\tau_{\text {abs }}$ : the reciprocal frequency for which the absorption curve has a maximum,

$\left.\tau_{\text {hf }} \lim _{\operatorname{def}} \frac{1}{\omega \rightarrow \infty} \frac{\chi^{\prime \prime}}{\chi^{\prime}-(1-F)}{ }^{6}\right)$.

Furthermore, explaining the experimental results with a distribution of relaxation times, a mean relaxation time $\bar{\tau}$ is defined 7 ). If the salt obeys the formulae of Casimir and Du Pré (1.6), all these quantities have the same value and equal $\tau_{s}$. In practice, however, they are different and their relation to $\tau_{\mathrm{S}}$ is unknown.

An important step to find the spin-lattice relaxation time has been taken by Eisenstein ${ }^{8}$ ). He computed the influence of a finite coefficient of heat conduction and of a finite heat capacity of a spherical sample, assuming the heat conductivity of the surroundings to be infinitely large. By adapting theoretical curves to experimental ones (varying $\tau_{\mathrm{s}}$ in the expressions for $\chi^{\prime}$ and $\left.\chi^{\prime \prime}\right)$ one finds a remarkable agreement between $\tau_{\mathrm{hr}}$ and the resulting value of $\tau_{\mathrm{s}}{ }^{4}$ ).

The aim of this paper is to calculate the influence of thermal properties of the surroundings on the differential magnetic susceptibility on the basis of the considerations of Casimir-Du Pré and Eisenstein. These properties are the coefficient of heat conduction, the heat capacity and a possible heat resistance between the surface of the sample and the surroundings. The calculations will be made for a spherical sample placed in a homogeneous infinitely large bath (e.g. liquid or gaseous helium).

2. A general expression for the differential magnetic susceptibility. We shall now derive a general expression for the differential magnetic susceptibility, taking into account the finite specific heat and coefficient of heat conduction of the lattice. Assumptions 2 and 3, mentioned in the preceding section under (1.4), hold with the following considerations. The basic formulae are:

$$
\begin{aligned}
& \mathrm{d} Q_{\mathrm{s}}=C_{H}\left(\frac{\partial T_{\mathrm{s}}}{\partial M}\right)_{H} \mathrm{~d} M+C_{M}\left(\frac{\partial T_{\mathrm{s}}}{\partial H}\right)_{M} \mathrm{~d} H, \\
& \frac{\mathrm{d} Q_{\mathrm{s}}}{\mathrm{d} t}=-\alpha\left(T_{\mathrm{s}}-T_{1}\right),
\end{aligned}
$$




$$
\begin{aligned}
& \mathrm{d} M=\left(\frac{\partial M}{\partial T_{\mathrm{s}}}\right)_{H} \mathrm{~d} T_{\mathrm{s}}+\left(\frac{\partial M}{\partial H}\right)_{T_{\mathrm{s}}} \mathrm{d} H, \\
& \frac{\partial}{\partial t} C_{1} T_{1}=\lambda_{1} V^{2} T_{\mathrm{l}}+P(x, y, z, t), \\
& P(x, y, z, t)=\alpha\left(T_{\mathrm{s}}-T_{1}\right) .
\end{aligned}
$$

(2.1) is related to the first law of thermodynamics for the spin system and, together with (2.2) and (2.3), it forms the basis for the theory of Casimir and Du Pré. (2.4) and (2.5) have been introduced by Eisenstein ${ }^{8}$ ); $C_{1}$ is the specific heat per volume of the lattice; $\lambda_{1}$ is the coefficient of heat conduction of the lattice, $P(x, y, z, t)$ is a heat source, which in general is a function of position and time. $T_{\mathrm{s}}$ and $T_{1}$ can be written in the form (cf. (1.1) and (1.2)):

$$
T_{\mathrm{s}}=T_{\mathrm{c}}+\theta_{\mathrm{s}} \operatorname{expi\omega t;} \quad T_{1}=T_{\mathrm{c}}+\theta_{1} \exp \mathrm{i} \omega t
$$

From (2.3) follows, with (1.1), (1.2) and (2.6) an expression for the reduced differential magnetic susceptibility:

$$
\chi=\frac{m_{0} / h_{0}}{(\partial M / \partial H)_{T_{\mathrm{c}}}}=1+\left(\frac{\partial H}{\partial M}\right)_{T_{\mathrm{c}}}\left(\frac{\partial M}{\partial T_{\mathrm{s}}}\right)_{H_{\mathrm{c}}} \frac{\theta_{\mathrm{s}}}{h_{0}} .
$$

Generally $\theta_{\mathrm{s}}$ will be a function of position. So we define the (measurable) mean susceptibility $\bar{\chi}$ :

$$
\bar{\chi}=\frac{1}{V} \int_{V} \frac{m_{0}}{h_{0}} \frac{1}{\left(\frac{\partial M}{\partial H}\right)_{T_{0}}} \mathrm{~d} \tau,
$$

$V$ is the volume of the sample, $\mathrm{d} \tau$ a volume element.

From (2.1), (2.2) and (2.3) we can derive a relation between $T_{1}$ and $T_{\mathrm{s}}$ :

$$
\begin{aligned}
& \frac{\partial}{\partial t} C_{H} T_{\mathrm{s}}=-\alpha\left(T_{\mathrm{s}}-T_{1}\right)+ \\
& \quad-\left[C_{H}\left(\frac{\partial T_{\mathrm{s}}}{\partial M}\right)_{H}\left(\frac{\partial M}{\partial H}\right)_{T_{\mathrm{s}}}+C_{M}\left(\frac{\partial T_{\mathrm{s}}}{\partial H}\right)_{M}\right]_{\frac{\partial}{\partial t} H} H
\end{aligned}
$$

and so (with (2.6)):

$$
\theta_{\mathrm{s}}-\left(1+\mathrm{i} \omega \tau_{\mathrm{s}}\right)^{-1}\left(\frac{\partial T_{\mathrm{s}}}{\partial H}\right)_{M_{\mathrm{c}}} h_{0} F \mathrm{i} \omega \tau_{\mathrm{s}}-\left(1+\mathrm{i}\left(\omega \tau_{\mathrm{s}}\right)^{-1} \theta_{1}=0 .\right.
$$

$\bar{\chi}$ can now be written in the form:

$$
\bar{\chi}=1-\frac{F \mathrm{i} \omega \tau_{\mathrm{s}}}{1+\mathrm{i} \omega \tau_{\mathrm{s}}}+\left(\frac{\partial M}{\partial T_{\mathrm{s}}}\right)_{H_{\mathrm{c}}} \frac{1}{\left(\frac{\partial M}{\partial H}\right)_{T_{\mathrm{c}}}} \frac{1}{h_{0}} \frac{1}{1+\mathrm{i} \omega \tau_{\mathrm{s}}} \frac{1}{V} \int_{V} \theta_{1} \mathrm{~d} \tau
$$

For $\theta_{\mathrm{l}}$ shall be derived a differential equation. 
From (2.4) and (2.5) we gret:

$$
\frac{\partial}{\partial t} C_{1} T_{1}=\lambda_{1} \nabla^{2} T_{1}+\alpha\left(T_{\mathrm{s}}-T_{1}\right)
$$

With the aid of (2.6) and (2.10) this equation can be transformed into:

$$
V^{2} \theta_{1}-L(\omega) \theta_{1}-M(\omega)=0 .
$$

In (2.13) the following abbreviations are used:

$$
\begin{aligned}
& \tau_{1}={ }_{\alpha}^{C_{1}}, \\
& L(\omega)=\frac{C_{1}}{\lambda_{1} \tau_{1}}\left[1+\mathrm{i} \omega \tau_{1}-\frac{1}{1+\mathrm{i} \omega \tau_{\mathrm{s}}}\right],
\end{aligned}
$$

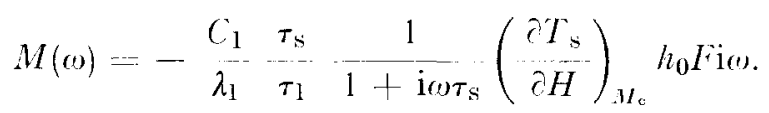

To determine $\bar{\chi}$ we must solve (2.13) with the correct boundary conditions. For a spherical sample in a homogeneous infinitely large bath, $\bar{\chi}$ can be expressed in terms of the value of $\theta_{1}$ on the boundary of the sample. In this case $\theta_{1}$ will be spherically symmetric. We define a new function of the spherical coordinate $r$ :

$$
\zeta_{1} \frac{r \theta_{1}}{M(\omega)}
$$

Now (2.11) and (2.13) can be rewritten in the form:

$$
\begin{aligned}
& \frac{\partial^{2} \zeta_{1}}{\partial r^{2}}-L(\omega) \zeta_{1}-r=0, \\
& \bar{\chi}=1-\frac{F i \omega \tau_{s}}{1+i \omega \tau_{s}}+\frac{C_{1} \tau_{s}}{\lambda_{1} \tau_{1}} \frac{I \ddot{i} \omega}{\left(1+\dot{\left.\mathrm{i} \omega \tau \tau_{s}\right)^{2}}\right.} \quad \frac{1}{V} \int_{l} \frac{\zeta_{1}}{r} \mathrm{~d} \tau
\end{aligned}
$$

The solution of (2.18) has to obey the boundary conditions:

$$
\zeta_{1}(0)=0, \quad \zeta_{1}\left(r_{0}\right)=\zeta_{\mathrm{B}} .
$$

Here $r_{0}$ is the radius of the sample; $\zeta_{\mathrm{B}}$ will be determined in section 3 . So an inhomogeneous differential eq. (2.18) with inhomogeneous boundary conditions (2.20) has to be integrated. For the solution we make use of the set of functions

$$
v_{n}(r)=\sqrt{\frac{2}{r_{0}}} \sin n \frac{\pi}{r_{0}} \cdot r \quad n=1,2,3 \ldots,
$$

which is an orthonormal set, complete for the solutions of (2.18) and (2.20). 
These functions $v_{n}(r)$ are eigenfunctions of the operator $\partial^{2} / \partial r^{2}-L(\omega)$ with eigenvalue $\mu_{n}$ :

$$
\mu_{n} \overline{\overline{\mathrm{def}}}-L(\omega)-\frac{n^{2} \pi^{2}}{r_{0}^{2}} .
$$

This leads to the following expression for $\zeta_{1}(\boldsymbol{r})$ :

$$
\zeta_{1}=\zeta_{\mathrm{B}} \cdot \frac{r}{r_{0}}+\sum_{n=1}^{\infty} \frac{1}{\mu_{n}} v_{n}(r) \int_{0}^{r_{0}} r\left[L(\omega) \frac{\zeta_{\mathrm{B}}}{r_{0}}+1\right] v_{n}(r) \mathrm{d} r
$$

For $\bar{\chi}$ then holds:

$$
\begin{aligned}
\bar{\chi}=1 & -\frac{F \mathrm{i} \omega \tau_{\mathrm{s}}}{1+\mathrm{i} \omega \tau_{\mathrm{s}}}+\frac{C_{1}}{\lambda_{1}} \frac{\tau_{\mathrm{s}}}{\tau_{1}} \frac{F \mathrm{i} \omega}{\left(1+\mathrm{i} \omega \tau_{\mathrm{s}}\right)^{2}} \times \\
\times & {\left[\frac{6}{\pi^{2}} \sum_{n=1}^{\infty} \frac{1}{n^{2}}-\frac{1}{\mu_{n}}+\frac{\zeta_{\mathrm{B}}}{r_{0}}+\frac{\zeta_{\mathrm{B}}}{r_{0}} L(\omega) \cdot \frac{6}{\pi^{2}} \sum_{n=1}^{\infty} \frac{1}{n^{2}} \frac{1}{\mu_{n}}\right] . }
\end{aligned}
$$

So the problem has been reduced to the determination of $\zeta_{\mathbf{B}}$. This will be done in the next section.

3. The influence of the thermal properties of the surroundings and of heat resistance at the surface of the sample. In this section we derive an expression for $\zeta_{\mathbf{B}}$, which depends on the coefficient of heat conduction of the surroundings and on a heat resistance on the surface of the sample.

There are two conditions for the energy flow through the surface:

$$
\begin{aligned}
& \lambda_{\mathrm{l}}\left(\nabla T_{\mathrm{l}}\right)_{r_{\mathrm{o}}}=\lambda_{\mathrm{g}}\left(\nabla T_{\mathrm{g}}\right)_{r_{0}}, \\
& \lambda_{\mathrm{l}}\left(\nabla T_{\mathrm{l}}\right)_{r_{\mathrm{o}}}=\frac{1}{R_{\mathrm{k}}}\left(T_{\mathrm{g}}-T_{\mathrm{l}}\right) r_{r_{0}} ;
\end{aligned}
$$

$\lambda_{\mathrm{g}}$ is the coefficient of heat conduction of the bath; $T_{\mathrm{g}}$ is the bath temperature; $R_{\mathbf{k}}$ is a heat resistance, which causes a temperature jump on the surface (at temperatures below the $\lambda$-point of liquid helium this will be the Kapitza resistance).

For $T_{\mathrm{g}}$ holds:

$$
\lambda_{\mathrm{g}} \nabla^{2} T_{\mathrm{g}}=\frac{\partial}{\partial t} C_{\mathbf{g}} T_{\mathrm{g}} ;
$$

$C_{\mathbf{g}}$ is the specific heat per volume of the bath.

Writing

$$
T_{\mathbf{g}}=T_{\mathrm{c}}+0_{\mathbf{g}} \exp i \omega t
$$

and

$$
\zeta_{\mathbf{g}}(r)=\frac{r \theta_{\mathbf{g}}}{\overline{\text { def }}}-
$$


we find

$$
\partial \gamma^{2} \zeta_{\mathrm{g}}-\mathrm{i} \omega \frac{C_{\mathrm{g}}}{\lambda_{\mathrm{g}}} \zeta_{\mathrm{g}}=0
$$

The general solution of (3.6) can be written:

$$
\zeta_{\mathrm{g}}=A \mathrm{e}^{\beta r}+B \mathrm{e}^{\beta r} \quad(A, B \text { constants), }
$$

with

$$
\beta=-(1+i) \sqrt{\frac{\omega C_{\mathrm{g}}}{2 \lambda_{\mathrm{g}}}} .
$$

It is obvious that $B$ has to be zero and so we have:

$$
\zeta_{\mathrm{g}}=A \cdot \mathrm{e}^{\beta r} \text {. }
$$

(3.1) and (3.2) can be expressed in terms of $\zeta_{1}$ and $\zeta_{\mathbf{g}}$ :

$$
\begin{aligned}
& \left(\frac{\partial}{\partial r} \zeta_{\mathrm{l}}\right)_{r_{0}}-\left(\begin{array}{c}
\zeta_{\mathrm{l}} \\
r_{0}
\end{array}\right)_{r_{0}}=\frac{\lambda_{\mathrm{g}}}{\lambda_{\mathrm{l}}}\left(\frac{\partial}{\partial r} \zeta_{\mathrm{g}}\right)_{r_{\mathrm{o}}}-\frac{\lambda_{\mathrm{g}}}{\lambda_{\mathrm{l}}}\left(\begin{array}{c}
\zeta_{\mathrm{g}} \\
r_{0}
\end{array}\right)_{r_{\mathrm{g}}}, \\
& \left(\frac{\hat{\partial}}{\partial r} \zeta_{\mathrm{l}}\right)_{r_{0}}-\left(\frac{\zeta_{1}}{r_{0}}\right)_{r_{0}}=\frac{1}{R_{\mathrm{k}} \lambda_{\mathrm{l}}}\left(\zeta_{\mathrm{g}}-\zeta_{1}\right)_{r_{0}} .
\end{aligned}
$$

By substituting (3.9) and (2.23) into (3.10) and (3.11) we have come to two lineair equations with two unknown variables $A$ and $\zeta_{\mathrm{B}}$. For $\zeta_{\mathrm{B}}$ it is finally found:

$$
\begin{aligned}
\frac{\zeta_{\mathbf{B}}}{r_{0}} & =\left[1-\frac{\lambda_{\mathrm{g}}}{r_{0}} R_{\mathrm{k}}\left(r_{0} \beta-1\right)\right] 2 \sum_{n=1}^{\infty} \mu_{n} \times \\
& \times\left[\left(r_{0} \beta-1\right)\left(2 \sum_{n=1}^{\infty} \frac{L(\omega)}{\mu_{n}} \frac{\lambda_{\mathrm{g}}}{r_{0}} R_{\mathrm{k}}-\frac{\lambda_{\mathrm{g}}}{\lambda_{1}}\right)-2 \sum_{n=1}^{\infty} \mu_{n} L(\omega)\right]^{1} .
\end{aligned}
$$

In the limiting case $R_{\mathrm{k}}=0, \lambda_{\mathrm{g}} \rightarrow \infty$ the expression for $\bar{\chi}$ (combination of (3.12) and (2.24)) must turn into the formula of Eisenstein ${ }^{8}$ ). However, the form of this expression, is quite different from that of Eisenstein. It can be shown that they are identical.

4. Discussion. In this section the result of the preceding sections will be applied to some experimental cases. The calculations are related to a spherical sample of CrK-alum, placed in (gaseous or liquid) helium and, if the value of the magnetic field $H_{\mathrm{c}}$ is not mentioned, this amounts:

$$
H_{\mathrm{c}}=179 \times 10^{3} \mathrm{~A} / \mathrm{m} \text {. }
$$

The following parameters characterize the sample:

$$
\left.\begin{array}{l}
r_{0}=8 \times 10^{-3} \mathrm{~m}, \quad C_{1}=15.07 \times T^{3} \mathrm{~J} / \mathrm{K} \cdot \mathrm{m}^{3} \\
\mu_{0} \mathrm{C}=109 \times 10^{-9} \mathrm{~J} \cdot \mathrm{K} / \mathrm{A}^{2} \cdot \mathrm{m}, \quad b / \mu_{0} \mathrm{C}=4.5 \times 10^{9} \mathrm{~A}^{2} / \mathrm{m}^{2} .
\end{array}\right\}
$$




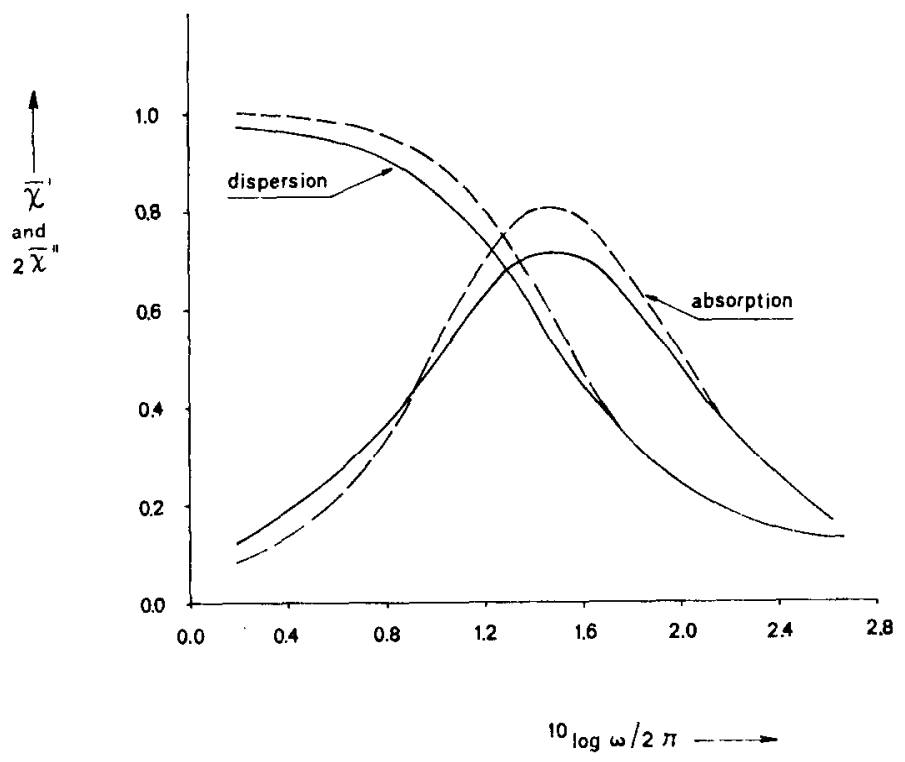

Fig. 1. Calculated dispersion and absorption curves according to formulae $(2.24)$ and $(3.12) ; \tau_{\mathrm{s}}=4 \times 10^{-3} \mathrm{~s}, \lambda_{\mathrm{I}}=1.5 \mathrm{~J} / \mathrm{s} \cdot \mathrm{K} \cdot \mathrm{m}$;

fully drawn: $C_{\mathrm{g}}=3.95 \times 10^{4} \mathrm{~J} / \mathrm{K} \cdot \mathrm{m}^{3}, \lambda_{\mathrm{g}}=2.2 \times 10^{-2} \mathrm{~J} / \mathrm{s} \cdot \mathrm{K} \cdot \mathrm{m}$, (values from Wilks $\left.{ }^{10}\right)$ );

dashed: $C_{\mathrm{g}}=4 \times 10^{5} \mathrm{~J} / \mathrm{K} \cdot \mathrm{m}^{3}, \lambda_{\mathrm{g}}=10^{20} \mathrm{~J} / \mathrm{s} \cdot \mathrm{K} \cdot \mathrm{m}$ (in agreement with the theory of Eisenstein).

$C$ is Curie's constant, $b$ is rclated to $C_{M}$ by the formula $C_{M}=b / T^{2} . C_{1}$ is given by $\left.\operatorname{Kapadnis}^{9}\right), b / \mu_{0} C$ follows from experimental dispersion curves, performed on a sample with parameters $(4.2)^{4}$ ).

A) The sample is placed in a liquid $\mathrm{He}$ bath, with temperature above the $\lambda$-point of liquid $\mathrm{He}$.

Calculations are carried out, the temperature of the sample being $T-$ $=3.021 \mathrm{~K}$, ignoring the heat resistance $\left(R_{\mathrm{k}}=0\right)$. From a comparison with the result of Eisenstein (this means in our formulation $\lambda_{\mathrm{g}} \rightarrow \infty$ ) follows a remarkable influence of a real value of $\lambda_{\mathrm{g}}$ on $\bar{\chi}$. In fig. I the real and imaginary part of the theoretical value of $\bar{\chi}=\bar{\chi}^{\prime}-\bar{\chi}^{\prime \prime}$ are drawn as functions of ${ }^{10} \log \omega / 2 \pi$ for both cases.

By varying $\tau_{\mathrm{s}}$ and $\lambda_{1}$ we tried to adapt a theoretical curve to an experimental one; the result is shown in fig. 2 .

Remark that $\tau_{\mathrm{abs}}$ and $\tau_{\text {disy }}$ (cf. sect. 1) differ considerably from $\tau_{\mathrm{s}}$ :

$$
\left.\tau_{\text {abs }}=4.4 \times 10^{-3} \mathrm{~s}, \quad \tau_{\text {disp }}=5.5 \times 10^{-3} \mathrm{~s}^{4}\right) .
$$

On account of these facts we conclude that the finite value of the coefficient of heat conduction in the He bath may have a measurable influence on $\bar{\chi}$. 


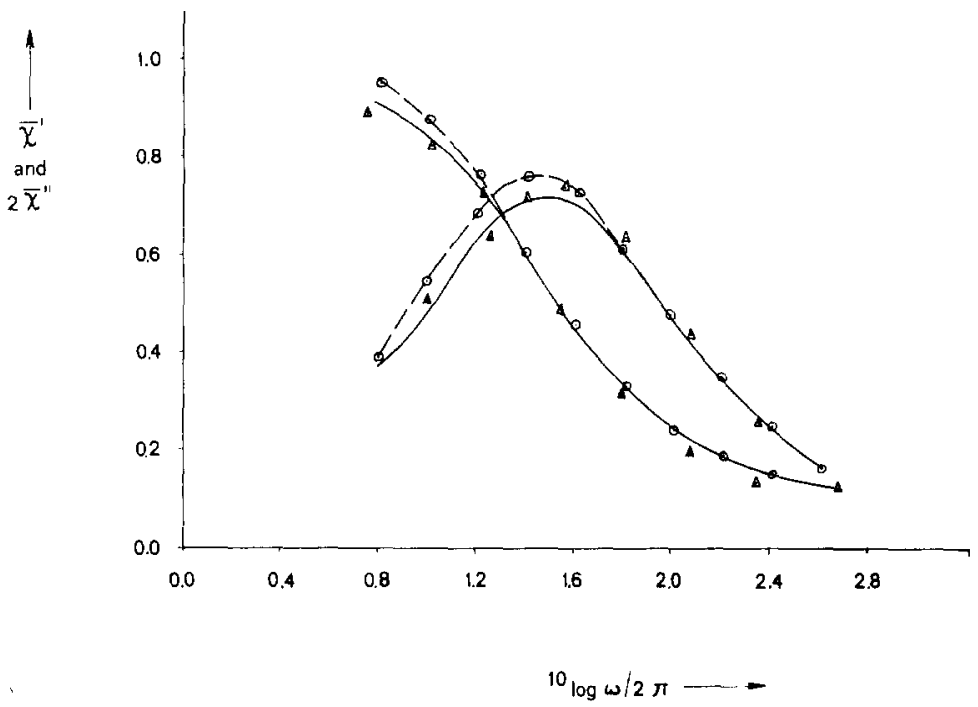

Fig. 2. Iispersion and absorption curves.

$\Delta$ : experimental curves, taken from a paper of v. d. Broek c.s. ${ }^{4}$ );

fully drawn: curves according to formulae (2.24) and (3.12) which have the best fit with experimental results;

$C_{\mathrm{g}}=3.95 \times 10^{4} \mathrm{~J} / \mathrm{K} \cdot \mathrm{m}^{3}, \lambda_{\mathrm{g}}=2.2 \times 10^{-2} \mathrm{~J} / \mathrm{s} \cdot \mathrm{K} \cdot \mathrm{m}$,

$\tau_{\mathrm{S}}=4 \times 10^{-3} \mathrm{~s}, \lambda_{\mathrm{I}}=1.5 \mathrm{~J} / \mathrm{s} \cdot \mathrm{K} \cdot \mathrm{m}$;

$O$ : the best fit according to the theory of Eisenstein (also from $\left.{ }^{4}\right)$ ); $\tau_{\mathrm{s}}=4 \times 10^{-3} \mathrm{~s}$, $\hat{\lambda}_{1}=0.9 \mathrm{~J} / \mathrm{s} \cdot \mathrm{K} \cdot \mathrm{m}$.

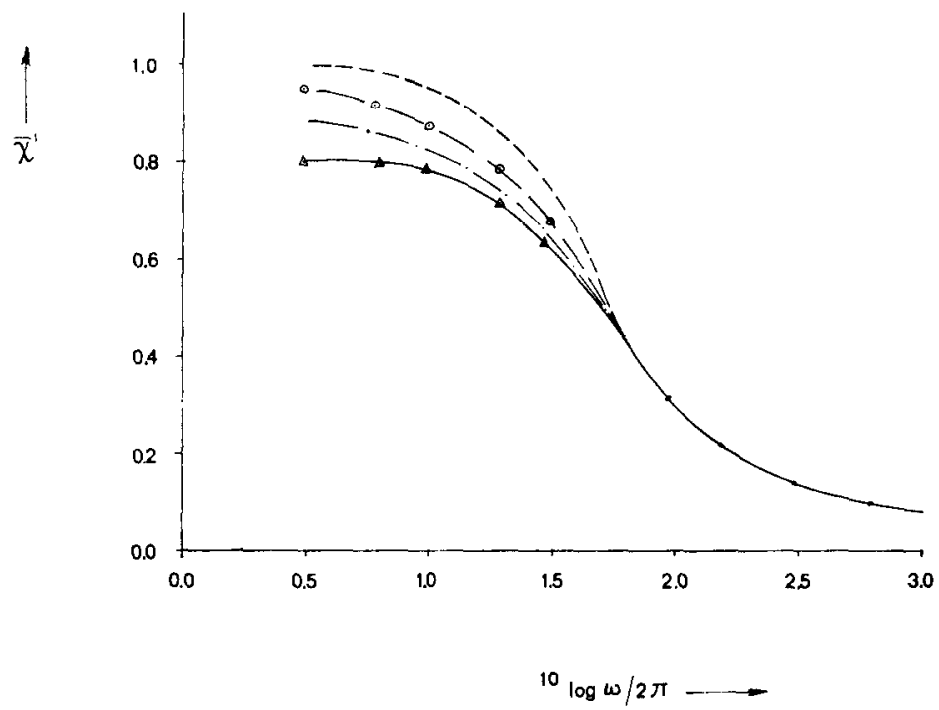

Fig. 3. Experimental dispersion curves of CrK-alum at various pressures of the surrounding helium gas.

$$
\begin{aligned}
& H=192 \times 10^{3} \mathrm{~A} / \mathrm{m}, \quad T=4.22 \mathrm{~K}, \quad \text { : } \quad p=34.8 \mathrm{~cm} \mathrm{Hg}, \\
& \triangle: \quad \text { isolated salt, } \quad \ldots: \quad p=76 \mathrm{~cm} \mathrm{Hg} \text {. } \\
& \text {-. - : } p=2.5 \mathrm{~cm} \mathrm{Hg} \text {, }
\end{aligned}
$$




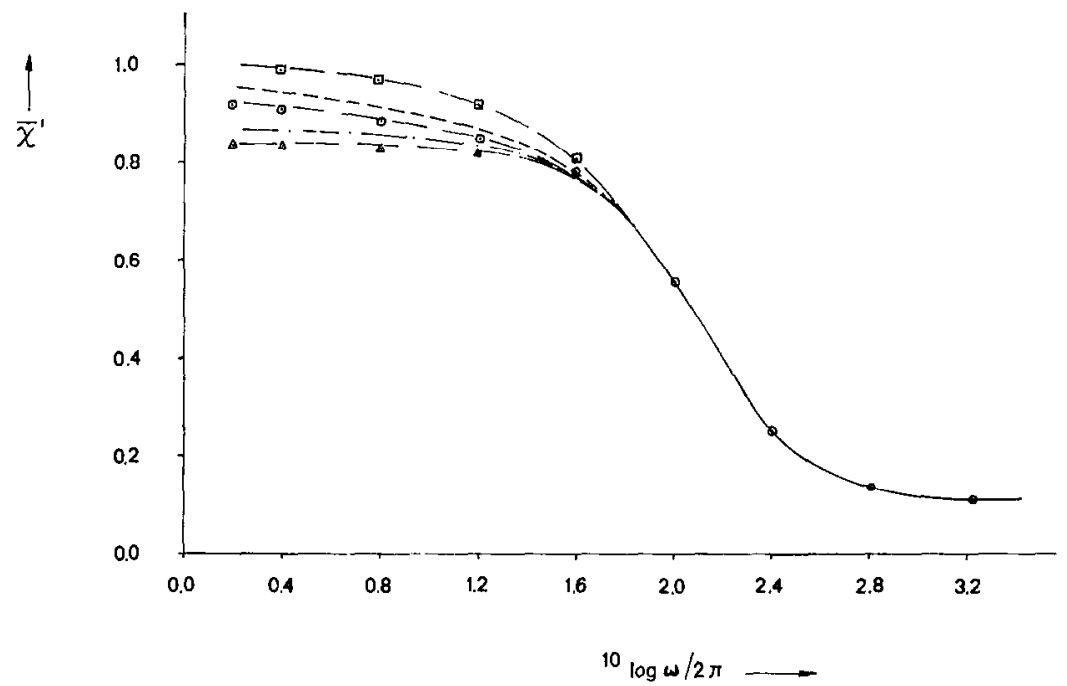

Fig. 4. Calculated dispersion curves of CrK-alum at various pressures of the surrounding helium gas.
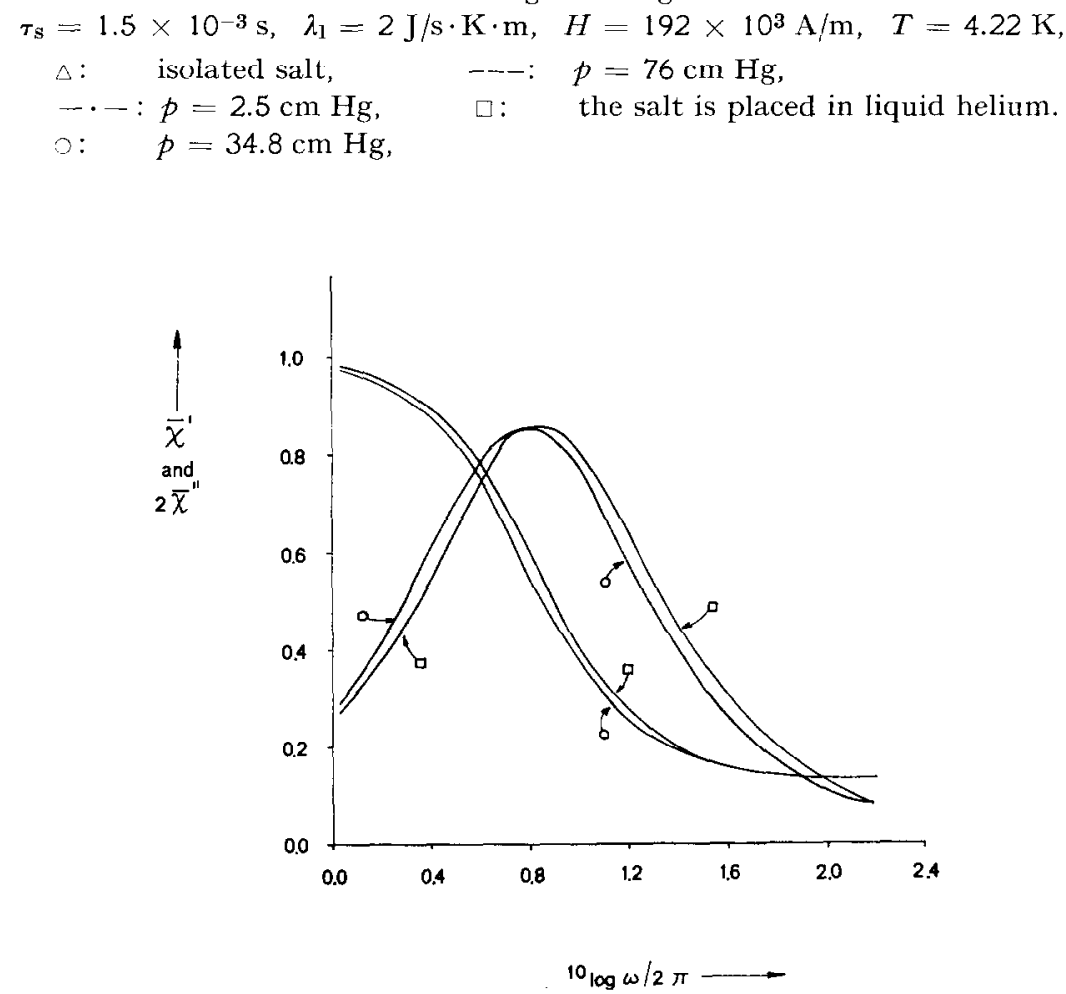

Fig. 5. Calculated absorption and dispersion curves of CrK-alum at different values
$\square \boldsymbol{R}_{\mathrm{k}}=0$, of $R_{\mathrm{k}}$.

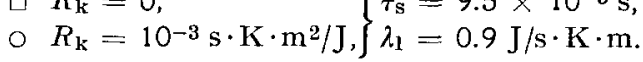




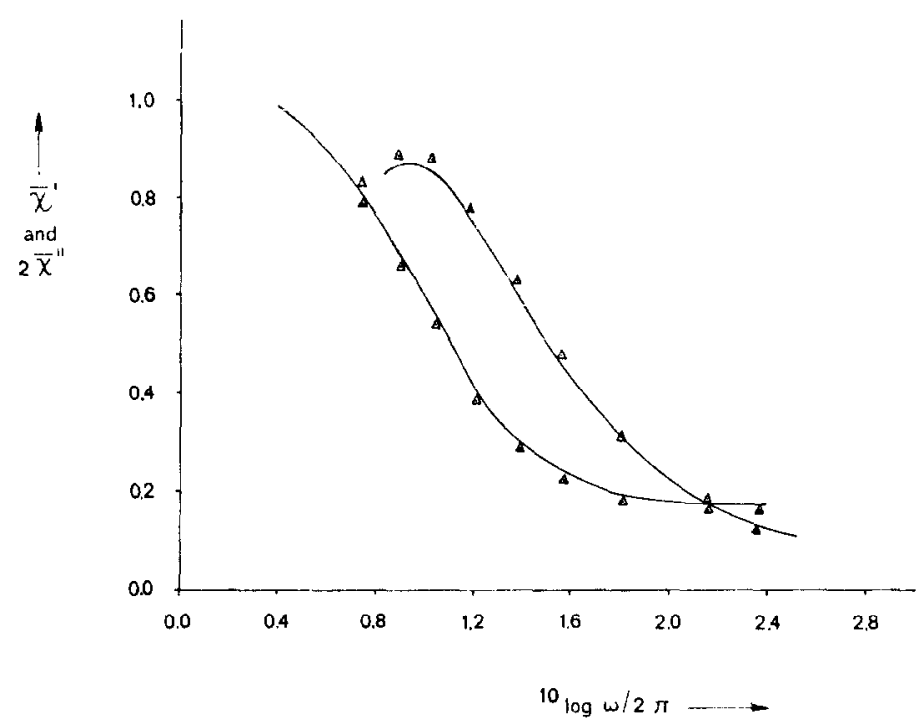

Fig. 6. Dispersion and absorption curves below the $\lambda$ point.

$\Delta:$ unpublished experimental curves of CrK-alum, measured at the Kamerlingh Onnes Iahoratory, Ieiden in relation to the investigations mentioned in ref. 4.

$H=179 \times 10^{3} \mathrm{~A} / \mathrm{m}, T=2.068 \mathrm{~K}$.

fully drawn: calculated curves, $R_{\mathrm{k}}=10^{-3} \mathrm{~s} \cdot \mathrm{K} \cdot \mathrm{m}^{2} / \mathrm{J}$,

$$
\tau_{\mathrm{s}}-9.5 \times 10^{-3} \mathrm{~s}, \lambda_{1}=0.7 \mathrm{~J} / \mathrm{s} \cdot \mathrm{K} \cdot \mathrm{m} \text {. }
$$

Note: variation of $\tau_{\mathrm{s}}$ and $\lambda_{1}$ within a range of respectively $10^{-4} \mathrm{~s}$ and 0.3 $\mathrm{J} / \mathrm{s} \cdot \mathrm{K} \cdot \mathrm{m}$ gives no better fit with the experimental curves.

B) The sample is placed in gaseous $\mathrm{He}$, at various pressures.

Experiments have been performed at the low temperature group of the Delft University of Technology on CrK-alum (not yet published) and on CoCs-tutton salt ${ }^{11}$ ); these show a remarkable influence of the pressure in the surrounding gas on the dispersion curves (fig. 3). We computed a series of dispersion curves at various $\lambda_{\mathrm{g}}{ }^{12}$ ) and $C_{\mathrm{g}}$, corresponding with different pressures (fig. 4). $C_{\mathrm{g}}$ has been calculated with the aid of experimental values of De Laet ${ }^{13}$ ) and with the equation of state of helium ${ }^{14}$ ).

The behaviour of these curves support the conclusion under A).

A remarkable experimental fact is that there is no difference between $\vec{\chi}$ measured at a sample in liquid $\mathrm{He}$ or at a sample in satureted He vapour (at the same temperature). Values of $C_{\mathrm{g}}$ and $\lambda_{\mathrm{g}}$ are different in both cases, and so are the calculated dispersion curves (fig. 4). This may be reason to suppose that there is some adsorption of He at the surface of the sample.

C) The sample is placed in a liquid $\mathrm{He}$ bath, at a temperature below the $\lambda$-point. We made calculations for $T=2.068 \mathrm{~K}$. From Beenakker ${ }^{15}$ ) and Pollack ${ }^{16}$ ) we estimated the Kapitza resistance to be $R_{\mathrm{k}}=10^{-3} \mathrm{~s} \cdot \mathrm{K} \cdot \mathrm{m}^{2} / \mathrm{J}$. 


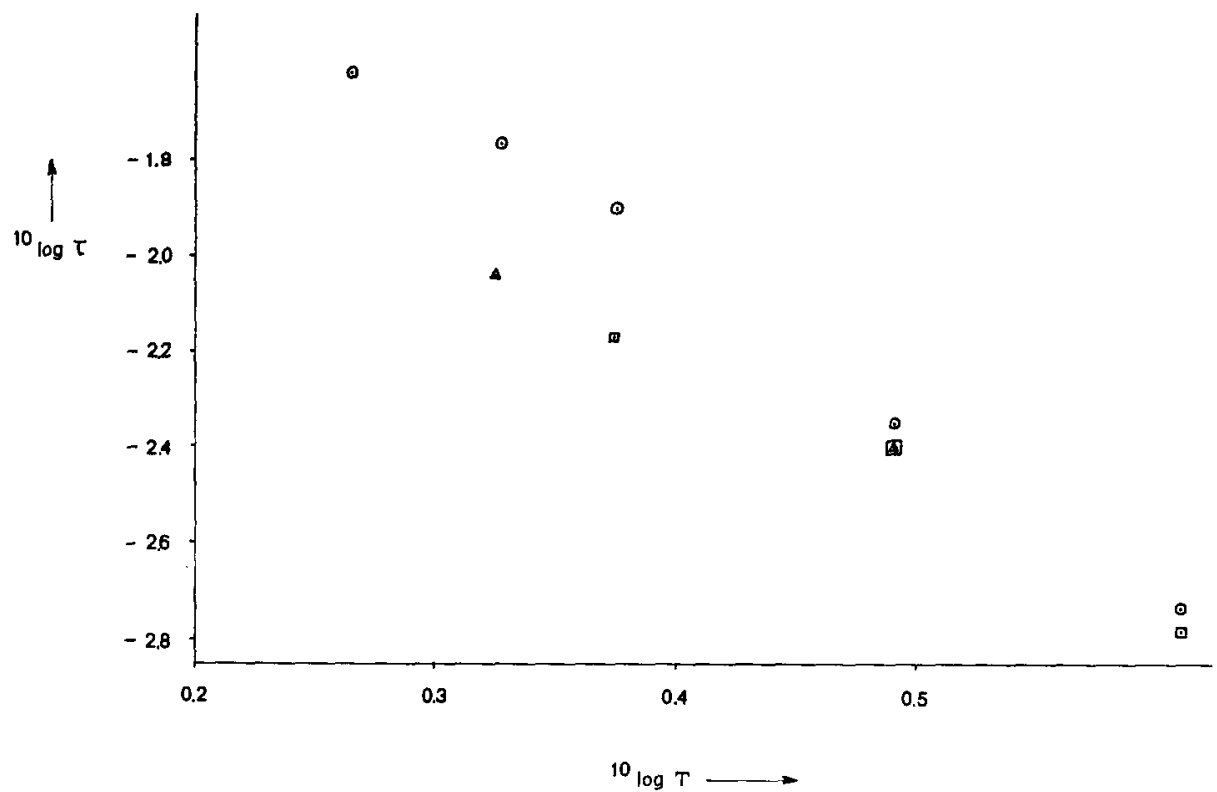

Fig. 7. Temperature dependency of $\tau$.

$O: \tau_{\text {abs }}$ and $\left.\tau_{\text {disp }}{ }^{4}\right)$.

$\left.\square: \tau_{\mathrm{hP}}{ }^{4}\right)$.

$\Delta$ : calculated values mentioned in the captions of figs. 2 and 6 .

That the Kapitza resistance may have a measurable influence on the magnetic behaviour of the sample is to be seen in fig. 5 .

Furthermore we tried to adapt theoretical curves to experimental ones by varying $\tau_{\mathrm{s}}$ and $\lambda_{1}$. The result is shown in fig. 6 .

Note: variation of $\tau_{\mathrm{s}}$ and $\lambda_{1}$ with respectively $10^{-4} \mathrm{~s}$ and $0.3 \mathrm{~J} / \mathrm{s} \cdot \mathrm{K} \cdot \mathrm{m}$ gives no better fit between the theoretical and experimental curves. On account of the following facts we conclude that the Kapitza effect may have a measurable influence on $\bar{\chi}$ : if we take $R_{\mathrm{k}}=0$, the best adaptation of the calculated curves to the experimental ones is reached for the values $\tau_{\mathrm{s}}=18 \times 10^{-3} \mathrm{~s}$ and $\lambda_{1}=10 \mathrm{~J} / \mathrm{s} \cdot K \cdot \mathrm{m}$. However, if for $R_{\mathrm{k}}$ the estimated value is taken $\left(R_{\mathrm{k}}=10^{-3} \mathrm{~s} \cdot \mathrm{K} \cdot \mathrm{m}^{2} / \mathrm{J}\right)$ the best fit is found at values $\tau_{\mathrm{s}}=9.5 \times 10^{-3} \mathrm{~s}$ and $\lambda_{1}=0.7 \mathrm{~J} / \mathrm{s} \cdot \mathrm{K} \cdot \mathrm{m}$. This $\tau_{\mathrm{s}}$ agrees rather well (fig. 7) with the temperature dependency of $\tau_{\mathrm{hf}}{ }^{4}$ ) and a computed $\tau_{\mathrm{s}}$ at $T=3.021 \mathrm{~K}$ (fig. 2). Mutatis mutandis the same holds for $\lambda_{1}$.

This investigation has been set up on account of the results of relaxation experiments at the Delft University of Technology. The authors are indebted to Professor Dr. B. S. Blaisse, Dr. G. J. C. Bots, Mr. J. H. H. Hülsmann and Mr. A. A. Los, who took part in the experiments, for the pleasant co-operation. 


\section{REFERENCES}

1) Hellwege, K. H., Kötzler, J. und Weber, G., Z. Phys. 217 (1968) 373. Amaya, K., Tokunaga, Y. and Haseda, T., Physica 40 (1969) 550.

Van Duyneveldt, A. J., Tromp, H. R. C. and Gorter, C. J., Physica 38 (1968) 205.

2) Casimir, H. B. G. and Du Pré, I. K., Physica 5 (1938) 507.

3) Kramers, H. C., Bij1, D. and Gorter, C. J., Physica 16 (1950) 65.

4) Van den Broek, J., Van der Marel, L. C. and Gorter, C. J., Physica 27 (1961) 661.

5) Van Vleck, J. H., Phys. Rev. 59 (1941) 724 and 730.

De Vries, A. J., Livius, J. W. M., Curtis, 1). A., Van Duyneveldt, A. J. and Gorter, C. J., Physica 36 (1967) 65.

Standley, K. J. and Vaughan, R. A., Electron Spin Relaxation Phenomena in Solids, Adam Hilger Ltd., London.

6) Benzie, R. J. and Cooke, A. H., Proc. Phys. Soc. London A 63 (1950) 201.

7) Fuoss, R. and Kirkwood, S. G., J. Amer. Chem. Soc. 63 (1941) 385.

Fang, P. H., Physica 24 (1958) 970.

8) Eisenstein, J., Phys. Rev. 84 (1951) 548.

9) Kapadnis, D. G., Physica 22 (1956) 159.

10) Wilks, J., The Properties of Liquid and Solid Helium, Clarendon Press Oxford, (1967).

11) Van der Marel, L. C., Bots, G. J. C. and Blaisse, B. S., Proc. Colloque Ampere, Grenoble (1968) 480.

12) Ubbink, J. B., Physica 13 (1947) 659.

13) De Laet, J., Verh. Kon. V1. Ac. v. Wetensch. XXI1, 1960, nr. 66.

14) Kilpatrick, J. E, Keller, W. E. and Hammel, E. F., Phys. Rev. 97 (1955) 9.

15) Beenakker, J. J. M., Taconis, K. W., Lynton, E. A., Dokoupil, Z. and Van Soest, G., Physica 18 (1952) 433.

16) Pollack, G. L., Rev. mod. Phys. 41 (1969) 48. 